\title{
Clinical value and feasibility of ISET in detecting circulating tumor cells in early breast cancer
}

\author{
Hanling Zeng $^{1 \#}$, Jordee Selvamanee Veeramootoo ${ }^{1 \#}$, Ge Ma ${ }^{1,2 \#}$, Yi Jiang ${ }^{1}$, Jingyi Wang ${ }^{1}$, Tiansong Xia ${ }^{1,2}$, \\ Xiaoan Liu ${ }^{1}$ \\ ${ }^{1}$ Department of Breast Surgery, The First Affiliated Hospital with Nanjing Medical University, Nanjing, China; ${ }^{2}$ Jiangsu Key Lab of Cancer \\ Biomarkers, Prevention and Treatment, Jiangsu Collaborative Innovation Center For Cancer Personalized Medicine, School of Public Health, \\ Nanjing Medical University, Nanjing, China \\ Contributions: (I) Conception and design: T Xia; (II) Administrative support: X Liu; (III) Provision of study materials or patients: G Ma, J Wang; (IV) \\ Collection and assembly of data: H Zeng, JS Veeramootoo; (V) Data analysis and interpretation: Y Jiang, JS Veeramootoo; (VI) Manuscript writing: \\ All authors; (VII) Final approval of manuscript: All authors. \\ \#These authors contributed equally to this work. \\ Correspondence to: Prof. Dr. Xiaoan Liu; Prof. Dr. Tiansong Xia. Department of Breast Surgery, The First Affiliated Hospital with Nanjing Medical \\ University, 300 Guangzhou Road, Nanjing 210029, China. Email: liuxiaoan@126.com; xiatsswms@163.com.
}

\begin{abstract}
Background: Patients with operable breast cancer have a better prognosis for recovery. However, once distant organ metastasis occurs, the chance of a long-term survival or a cure is limited. The collection and counting of circulating tumor cells (CTCs) by reliable detection techniques are of increasing importance in the diagnosis of early metastasis and prognosis of disease progression. Isolation by size of epithelial tumor cells (ISET) has the advantage of simplicity of operation and high homogeneity. It is practical for large-scale clinical detection showing cell abundance. The value of ISET in the detection of circulating breast cancers in the blood has not been determined. The purpose of this study is to explore the feasibility of applying ISET to detect CTCs by determining the detection rate of ISET in operable breast cancer and by evaluating the correlation between detection rate, cell count and clinical factors such as molecular typing and pathological staging.
\end{abstract}

Methods: The experiment included 193 breast cancer patients who were diagnosed by core needle biopsy before the operation. $10 \mathrm{~mL}$ of venous blood was collected from the patients preoperatively, and CTCs in their blood samples were counted and analyzed by ISET.

Results: Patients were divided into groups according to pathology and immunohistochemistry. The overall detection rate of CTCs by ISET was $41.24 \%$. The detection rate, the number of overall CTCs and the average number of CTCs in each group were analyzed individually. No significant differences were observed between the different groups.

Conclusions: Although ISET has a relatively good detection rate for circulating breast cancer cells, it fails to provide more information on pathological staging, molecular classification and so forth.

Keywords: Isolation by size of epithelial tumor cells (ISET); circulating tumor cells (CTCs); breast cancer

Submitted Dec 01, 2019. Accepted for publication Jun 03, 2020.

doi: $10.21037 /$ tcr-19-2662

View this article at: http://dx.doi.org/10.21037/tcr-19-2662 


\section{Introduction}

Breast cancer is the most common malignancy worldwide and remains the leading cause of cancer death in women. It accounts for $25 \%$ of all cancers and $15 \%$ of cancer deaths (1). Although non-metastatic breast cancer patients can be effectively cured by surgery, chemotherapy, radiotherapy, endocrine therapy, targeted therapy, and other comprehensive treatment, distant organ metastasis is common and incurable (2). Breast cancer is considered to be a systemic disease due to early spread with small tumor burden. Early detection of micro-metastasis derived from minimal residual lesions or disseminated tumor cell (DTC) or circulating tumor cell (CTC) in the blood cannot be achieved by high-resolution diagnostic imaging technologies (3). Studies have shown that tumor cells can be identified in bone marrow (DTCs) and peripheral blood (CTCs) in patients with non-metastatic early breast cancer $(4,5)$. Additionally, minimal residual lesions of breast cancer can be evaluated in bone marrow, and patients with positive DTCs have a poor prognosis $(6,7)$. Furthermore, monitoring DTCs in bone marrow after adjuvant therapy has demonstrated clinical significance (8). However, repeated bone marrow aspiration under local anesthesia is difficult, which makes the detection of DTCs tedious, painful and invasive. CTCs that can be obtained via peripheral blood sampling have received more clinical attention due to good tolerance, minimally invasiveness and reproducibility of the procedure.

The prognostic value of detection CTCs in patients with early breast cancer has been reported $(9,10)$. The increase in the number of CTCs correlates with lower survival rates, and the persistent existence of CTCs during treatment is indicative of poor response to therapy. Detection of CTCs from blood tests is a reliable tool to assess the risk of recurrence and metastasis. CTC count, as a prognostic biomarker for distant metastasis in early breast cancer patients will play an increasingly important role in breast cancer management.

Advances in detection methods have made the detection of CTCs more reliable (11). The detection process mainly includes two steps: enrichment and labeling identification. Due to the rarity of CTCs, an effective enrichment method is the key and a technical challenge. At present, different CTC detection methods have different sensitivity, specificity, reliability, cost-effectiveness and limitations. In the exploration of different detection methods (12-16), we found Isolation by size of epithelial tumor cells (ISET) is a CTC enrichment method based on the size of epithelial tumor cells. One advantage of ISET is its capability of enriching a large number of CTCs or circulating tumor microemboli (CTM) from the peripheral blood of breast cancer patients.

The main purpose of this study was to test the effectiveness of ISET in detecting CTCs in patients with operable breast cancer, and subsequently to determine the correlations between CTCs count and molecular classification and pathological staging, as well as CTCs as biomarkers for the prognosis of early stage breast cancer. We present the following article in accordance with the STROBE reporting checklist (available at http://dx.doi. org/10.21037/tcr-19-2662).

\section{Methods}

\section{General information}

A total of 193 breast cancer patients who underwent surgery in the Breast Diseases Department of the First Affiliated Hospital with Nanjing Medical University (February 2019 to May 2019) were included in this study. Specific inclusion criteria: (I) All patients had confirmed diagnosis of breast cancer by preoperative biopsy (puncture, etc.), including ductal carcinoma in situ (DCIS), invasive ductal carcinoma (IDC), invasive lobular carcinoma (ILC), mucinous adenocarcinoma and so on. (II) Preoperative ultrasonography/mammography/magnetic resonance imaging (MRI) evaluation showed that the mass was $\leq 5 \mathrm{~cm}$ and enlarged axillary lymph nodes $\leq 9$. (III) Visceral metastasis was excluded by preoperative chest CT and abdominal CT/ultrasonography. (IV) There was no obvious abnormality in biochemistry, blood routine, and ECG before operation. (V) No history of taking special medications before operation. (VI) Patients who received neoadjuvant chemotherapy prior to surgery were excluded. (VII) No previous history of a malignant tumor. (VIII) Surgical methods include breast-conserving/total mastectomy + sentinel lymph node biopsy and modified radical mastectomy. The trial was conducted in accordance with the Declaration of Helsinki (as revised in 2013). The study was approved by Institutional Review Boards of the First Affiliated Hospital with Nanjing Medical University (NO.: 2017-SR-171) and informed consent was taken from all the patients. 


\section{Methods}

\section{Peripheral blood samples}

Peripheral blood samples were taken one hour before surgery from the median cubital vein and mixed with K2EDTA. The first $2.5 \mathrm{~mL}$ of blood was discarded to prevent epithelial cell contamination, and the remaining $7.5 \mathrm{~mL}$ were treated immediately (within 2 hours).

\section{ISET assay}

ISET assay was performed as described earlier by Vona et al. (17). Briefly, first, the blood $(7.5 \mathrm{~mL})$ was collected into the EDTA tube (Beckton Dickinson, NJ, USA) and divided into two equal samples of $0.75 \mathrm{~mL}$ each within 4 hours. Each small sample was diluted at 1:10 with the red blood cell dissolution buffer (Rarecell Diagnostics, Paris, France) and loaded into a separate hole in the ISET filtration module (Youzhiyou, Wuhan, China), which consists of a 10-hole plastic liquid storage tank located on top of a polycarbonate film with a cylindrical $8 \mathrm{~mm}$ hole. The blood sample was filtered by connecting the module to the ISET device and applying a mild adjustable suction. This process produced 10 discontinuous $6 \mathrm{~mm}^{2}$ membrane points on which cells of $0.75 \mathrm{~mL}$ whole blood were deposited. The membrane was stored at $-20{ }^{\circ} \mathrm{C}$. Analysis was conducted according to the manufacturer's protocol. Cells were classified as circulating tumor cells when they meet more than four of the following criteria: (I) significantly enlarged nucleus ( $>2-3$ calibrated aperture); (II) the high nucleocytoplasmic ratio (ratio $>0.8$ ); (II) deep and uneven staining; (IV) irregularity of the nuclear membrane; (V) increased number of heteronuclear cells (ratio >0.5); (VI) lateral displacement of nuclear chromatin or presence of large nucleolus; (VII) abnormal mitotic pattern. Cells without cytoplasm are not analyzed.

\section{Detection of CTCs}

Immunofluorescence staining was used to confirm the differentiated cluster CD45. The expression of CD45 was used to distinguish between CTCs and leukocytes, especially megakaryocytes and large monocytes. At room temperature $\left(18-26^{\circ} \mathrm{C}\right), 200 \mu \mathrm{L}$ paraformaldehyde $(2 \%)$ was added to the filter and cells were fixed for 5 minutes. Thereafter, cells were washed with PBS 3 times, with each wash for 2 minutes. $200 \mu \mathrm{L}$ methanol was then added to the filter and left to rest for 1 minute. Filter membrane was removed and placed onto one side of the glass slide, left to dry at room temperature for 4-5 minutes, and transferred to the center of the glass slide, where it was fixed with $2 \mu \mathrm{L}$ adhesive [(transparent reagent; catalogue No. BA-7002B); (mountant; catalogue No. BA-7004); Baso Biotechnology, Wuhan, China]. A circle was drawn around the filter membrane with a PAP pen. The sample was then treated with $200 \mu \mathrm{L} 0.5 \%$ Triton X-100 for 5 minutes and washed with PBS for 3 times with 2 minutes each time. Thereafter, $100 \mu \mathrm{L} 10 \%$ goat serum was added to the filter membrane (Jackson ImmunoResearch Europe, Newmarket, UK) and placed at room temperature for 30 minutes with the excess serum removed. The sample was incubated with $100 \mu \mathrm{L}$ primary antibody (anti-CD45; catalogue No. SC-70699; Santa Cruz Biotechnology, Dallas, TX) diluted to 1:500 and $1: 200$ with $10 \%$ goat serum, respectively at $4{ }^{\circ} \mathrm{C}$ overnight. Samples were washed with PBS for 3 times with 3 minutes for each wash, and $10 \%$ goat serum was added to dilute $100 \mu \mathrm{L}$ secondary antibody (Alexa Fluor 488-conjugated goat anti-rat; catalogue No. A11006; Thermo Fisher Technology, Waltham, MA, USA) to 1:500, and the slides were incubated with the secondary antibody at room temperature for 50 minutes. The slides were rinsed with PBS for 3 times with 2 minutes of each wash and the film was sealed with DAPI. Observation was done with a fluorescence microscope (magnification 40 times). After the slide images were taken, Giemsa staining was performed and compared with the results of immunofluorescence. Glass slides were dyed at room temperature with $100 \mu \mathrm{L}$ diff A (Eosin; catalogue No. YZY-CTC-p100; Youzhiyou, Wuhan, China) for 1 minute. The slides were rinsed with PBS for 1 minute and $100 \mu \mathrm{L}$ diff B (Methylthioninium Chloride; catalogue No. YZY-CTC-p100; Youzhiyou, Wuhan, China) was added for 90 seconds at room temperature. The glass slides were then washed with deionized water for 30 seconds each time and dried at $50{ }^{\circ} \mathrm{C}$ for 30 minutes. The glass slides were then dried for $1 \mathrm{~h}$ at $50{ }^{\circ} \mathrm{C}$ using a permanent installation medium (Baso UltraClear Advanced Mounting Resin; catalogue No. BA-7004; Baso Biotechnology, Wuhan, China). Finally, the cells were observed and counted with an optical microscope (magnified 40 times).

\section{Statistical analyses}

SPSS25.0 software was used for statistical analysis. The 
number of CTC in different groups was expressed by mean \pm standard deviation, the total data was tested by independent sample T-test, the data in the group were analyzed by single-factor analysis of variance and corrected by Turkey test, and the counting data were expressed as rate (\%). All $\mathrm{P}$ values were double tails, and $\mathrm{P}<0.05$ was considered statistically significant.

\section{Results}

\section{Clinicopathological parameters of the patients}

A total of 193 patients were enrolled between February 2019 and May 2019, including 1 case of male breast cancer and 192 cases of female breast cancer, aged between 2879 years. All patients underwent surgical resection (tumor resection without pathological residue) as the first-line treatment. Baseline patient clinical data included age, gender, pathological type, histological grade, tumor (T)

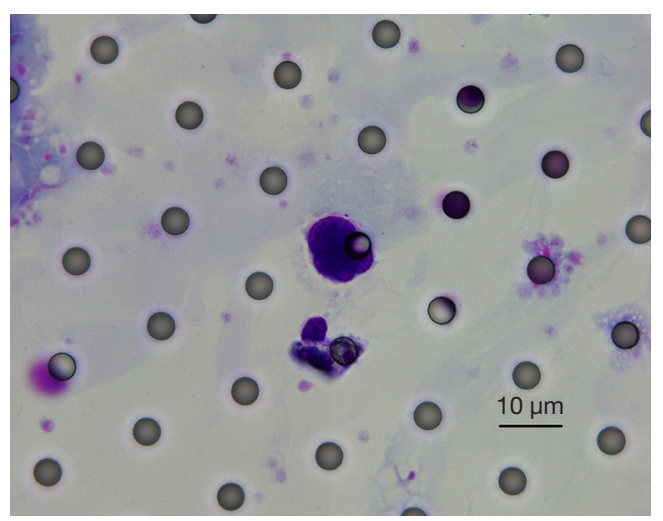

Figure 1 Detection of circulating tumor cells by ISET (isolation by size of epithelial tumor cells). Circulating tumor cells observed under a microscope: enlarged nucleus (about 3 calibrated aperture), thickened nuclear membrane, a high nuclear quality, irregular nucleoli shape, deep and uneven nuclear staining, and large amounts of cytoplasm in blue. stage, lymph node metastasis, hormone receptor status, Her-2 status, Ki-67 status, and CK5/6 status.

\section{Overall detection of CTCs}

Before the surgical resection, the total detection rate of CTCs was $40.93 \%$, the total number of CTCs was 189 , and the average number of CTCs was 0.979. Figure 1 shows a morphological analysis of CTCs by Giemsa staining, Diff-Quik staining (magnified 1000 times) and separated by CTCBiopsy ${ }^{\circledR}$ : enlarged nucleus (about 3 calibrated aperture), thickened nuclear membrane, a high nuclear content, irregular nucleoli shape, deep and uneven nuclear staining, and large amounts of cytoplasm in blue. However, we did not detect CTM in the blood of this cohort.

\section{Detection of CTCs in different groups}

\section{Pathological types with detection of CTCs in breast cancer}

Among the 193 patients, there were 167 cases of IDC, 9 cases of DCIS, 8 cases of ILC, 4 cases of mucinous adenocarcinoma, 3 cases of DCIS with invasion, 1 case of invasive micropapillary carcinoma and 1 case of invasive carcinoma. Three cases of DCIS with invasive and 1 case of invasive micropapillary carcinoma were included in IDC for the analysis. Due to the lack of other cases, 1 case of invasive carcinoma was excluded from the analysis. In 171 cases of IDC, the detection rate, total cell number and the average cell number were $40.94 \%, 158$ and 0.924 , respectively. The detection rate, total cell number and the average cell number of the ILC group were 50\%, 10 and 1.25, respectively. The detection rate, total cell number and the average cell number of the DCIS group were $33.33 \%, 9$ and 1 , respectively. The detection rate, total cell number and the average cell number of mucinous adenocarcinomas were $50 \%, 10$ and 2.5 respectively. There were no statistically significant differences in the detection of CTCs among the

Table 1 Pathological types with detection of circulating tumor cells in breast cancer

\begin{tabular}{lcccc}
\hline Pathological types & Patients & Positive rate (\%) & Mean \pm standard deviation & Total circulating tumor cells \\
\hline Invasive ductal carcinoma & 171 & 40.94 & $0.924 \pm 1.734$ & 158 \\
Invasive lobular carcinoma & 8 & 50.00 & $1.250 \pm 1.642$ & 10.3287 \\
Ductal carcinoma in situ & 9 & 33.33 & $1.000 \pm 1.766$ & 9 \\
Mucinous carcinoma & 4 & 50.00 & $2.500 \pm 1.647$ & 10 \\
\hline
\end{tabular}


Table 2 Pathological staging with detection of circulating tumor cells in breast cancer

\begin{tabular}{|c|c|c|c|c|c|}
\hline Pathological staging & Patients & Positive rate (\%) & Mean \pm standard deviation & Total circulating tumor cells & $P$ value \\
\hline$\| \mathrm{A}$ & 70 & 44.29 & $1.200 \pm 1.746$ & 84 & \\
\hline IIB & 23 & 39.13 & $0.826 \pm 1.773$ & 19 & \\
\hline IIIA & 14 & 35.71 & $0.571 \pm 1.650$ & 8 & \\
\hline
\end{tabular}

Table 3 Histological grading with detection of circulating tumor cells in breast cancer

\begin{tabular}{lccccc}
\hline Histological grading & Patients & Positive rate (\%) & Mean \pm standard deviation & Total circulating tumor cells & P value \\
\hline II & 75 & 35.14 & $0.892 \pm 1.744$ & 66 & 0.7264 \\
III & 77 & 44.16 & $1.000 \pm 1.734$ & 77 \\
II-III & 13 & 38.46 & $1.231 \pm 1.806$ & 16 \\
\hline
\end{tabular}

four groups of different pathological types of breast cancer (Table 1, P>0.05).

\section{Pathological staging with detection of CTCs in breast cancer}

Based on the seventh edition of NCCN guidelines, the survey population was divided into stage I, IIA, IIB, IIIA, IIIC and 0 (DCIS, as shown above) according to the number of lymph node metastasis, mass size and the presence or absence of distant metastasis (distant metastasis was excluded when 193 patients were recruited). Eleven patients who were unable to reassess the size of the mass in our hospital after mass resection were also excluded. The detection rate, total number and average of CTCs in 56 patients with stage I were $37.5 \%, 47$ and 0.839 , respectively. The detection rate, total number and average of CTCs in 70 patients with IIA were $44.29 \%, 84$ and 1.2 , respectively. The detection rate, total number and average of CTCs in 23 patients with IIB stage were $39.13 \%, 19$ and 0.826 , respectively. Furthermore, the detection rate, total number and average of CTCs in 14 patients with IIIA stage were $35.71 \%, 8$ and 0.571 , respectively. The detection rate, total number and average of CTCs in 10 patients with IIIC stage were $50 \%, 14$ and 1.4, respectively. There were no statistically significant differences in the detection of CTCs between the six pathological staging (Table 2, $\mathrm{P}>0.05$ ).

\section{Histological grading with detection of CTCs in breast cancer}

Special types of invasive carcinoma and ungrouped invasive carcinoma had no histological grade and were removed. DCIS cases were also excluded. The remaining cohort was divided into I grade, II grade, III grade and II-III grade according to the histological grade of invasive ductal carcinoma. There was only one patient in the Grade I group whereby one circulating tumor cell was detected, which was excluded as a special case. The detection rates of CTCs in the other three groups were $35.14 \%, 44.16 \%$, and $38.46 \%$, respectively. The total number of cells was 66,77 and 16 , and the average number of cells was $0.892,1$ and 1.231 , respectively. There were no significant differences in the detection of CTCs among the three histological groups. (Table 3, $\mathrm{P}>0.05$ ).

\section{Molecular typing with detection of CTCs in breast cancer}

Nine patients with DCIS and 1 patient whose subsequent immunohistochemical test was not conducted were excluded. According to the status of hormone receptor, her-2 and Ki67, the remaining cohort was divided into Luminal A group, Luminal B group, her-2 overexpression group, and triple-negative group. The detection rate was $44.44 \%, 41.18 \%, 40.74 \%, 42.86 \%$, respectively; the total 
Table 4 Molecular typing with detection of circulating tumor cells in breast cancer

\begin{tabular}{|c|c|c|c|c|c|}
\hline Molecular subtyping & Patients & Positive rate (\%) & Mean \pm standard deviation & Total circulating tumor cells & $P$ value \\
\hline Luminal B & 119 & 41.18 & $1.025 \pm 1.736$ & 122 & \\
\hline Her-2 overexpression & 27 & 40.74 & $0.888 \pm 1.750$ & 24 & \\
\hline Triple-negative & 28 & 42.86 & $1.071 \pm 1.616$ & 30 & \\
\hline
\end{tabular}

cell number was $4,122,24,30$, and the average cell number was $0.444,1.025,0.888,1.071$, respectively. There were no significant differences in the detection of circulating tumor cells between the four histological groups (Table 4, $\mathrm{P}>0.05$ ).

\section{Discussion}

The commonly used enrichment methods of CTCs include immunomagnetic, density gradient and filtration. CellSearch (18-20) relies on cell surface-specific antigen affinity capture. Although it is one of the most sensitive indirect methods in CTC detection (21) and has gained U.S. Food and Drug Administration's approval for the followup of patients with breast, colonic and prostate metastasis, this technique has some limitations, such as, false-positive selection may occur due to the expression of epithelial markers in non-epithelial cells. Similarly, circulating tumor markers that are not recognized by EpCAM antibodies are omitted as falsely negative. Therefore, the specificity of CellSearch is controversial (22-25). The density gradient centrifugation using Ficoll-Hypaque stratified solution depends on cellular physical specificity. CTCs are easily lost due to cell migration to plasma layers or cell aggregation. In addition, in order to prevent the mixing of different layers, the centrifugal step must begin as soon as possible. Moreover, the stratified solution is toxic which may affect the viability of cells. Although the density gradient method does not depend on the existence of specific markers and is considered to be a method for enriching all types of circulating tumor cells, its specificity raises concerns $(26,27)$. Although RT-PCR has been widely used in the past few years (28), the extraction of RNA destroys the integrity of cells (29) and hinders the analysis of cell morphology and phenotype, and the semi-quantitative nature of PCR prohibits the accurate estimation CTCs in the blood. The filtration method used in our study, ISET, is to separate CTCs based on the size of epithelial tumor cells, which is a method of direct enrichment of epithelial cells according to the characteristics of physical size. White blood cells and epithelial cells were separated by $8 \mu \mathrm{m}$ pore filter (17). Nucleated cells with positive expression of cytokeratin (CK) 8/18/19 but negative expression of white blood cell specific marker CD45 are CTCs.

ISET was the first method to allow direct filtration of peripheral blood, by avoiding immunomagnetism and flow cytometry cell separation $(30,31)$ to minimize cell loss. In fact, one of the most distinct advantages of ISET is that it can keep the isolated epithelial cells intact for morphological analyzes.

ISET was used to detect CTCs in the blood of 193 patients with operable breast cancer, with an overall detection rate of $40.93 \%$. According to few recent studies, the preoperative detection rate of CTCs in operable patients with esophageal cancer using ISET under similar inclusion criteria was $52.7 \%$ (32), for non-small cell lung carcinoma by ISET was $49.52 \%$, which was higher than the $39 \%$ detection rate of CellSearch (33). Out CTC detection rate in operable breast cancer patients by ISET is comparable the above-mentioned studies. The results showed the consistency of ISET for different malignancies which suggest that it may be used broadly.

A recent report showed that positive CTC count is associated with cancer risk, low CTC count $(<3 / \mathrm{mL})$ is associated with mild malignancy, and $3-20 \mathrm{CTC} / \mathrm{mL}$ is associated with moderate malignancy. High CTC count ( $>20 / \mathrm{mL}$ ) is associated with a higher risk of malignancy, recurrence, and metastasis (30). However, it is not the case in our study. After grouping 193 patients according to pathological type, histological grade, pathological staging or molecular classification, we found that there were no correlations between the detection rate, the total number and the average number of CTCs and the above clinical parameter. Similar results were observed in CTCs isolated by ISET in operable esophageal cancer (32). In another prospective clinical trial, CellSearch was used to detect CTCs in 115 patients with non-metastatic breast cancer 
diagnosed as operable or locally advanced breast cancer. CTCs were detected by CellSearch in $23 \%$ of patients, but only $10 \%$ of patients had $>1 / 7.5 \mathrm{~mL}$ circulating tumor cells in their blood. Subsequent studies have shown that even if only one CTC is detected, it is also related to the development of metastasis in the future, which indicates that the detection of CTCs does not need a threshold, and the metastasis efficiency of CTCs is higher than that of previous cognition (34). Therefore, we come to the conclusion that the detection of CTCs by ISET cannot provide more information on pathological staging and molecular classification, but can provide a more convenient, rapid and economical prognosis for the development of distant metastasis.

Current study also included a case of male breast cancer in which circulating tumor cells were detected. Male breast cancer is a rare malignant tumor, accounting for $0.2-1.5 \%$ of all male cancers and about $1 \%$ of breast cancer. Male breast cancer has a poor prognosis, but if it is detected early and treated promptly, the prognosis of treatment tends to be the same as that of women with breast cancer. The detection rate of CTCs in the whole male breast cancer population were higher than those in the female population with the same pathological type, pathological staging, and molecular classification, thus providing more help for the early diagnosis of male breast cancer. Nevertheless, due to the limited sample size at present, the identification of this type of cancer is still inadequate. This suggests that ISET may have better applicability and higher clinical values in male breast cancer.

The 193 patients have been compared in clinical parameters including most of the breast cancer pathology types (IDC, DCIS, ILC, mucinous adenocarcinoma, DCIS with invasive, invasive micropapillary carcinoma) and all the molecular classification and pathological staging of breast cancer. ISET has been evaluated comprehensively, but due to the limited sample size and large variations in case numbers among different groups, most subgroups can only observe the trend. Future investigations with large sample size and more even case number distribution of all analysis groups are required. In addition, because of the long-estimated survival time of breast cancer, this study is far from reaching the follow-up node. The prognostic value of this method in the preoperative detection of early breast cancer cannot be assessed immediately. According to the previous research experience, the detection efficiency of our previous data is better than most positive capture methods, suggesting that it still has great potential value in prognosis.
The observations of this study have provided evidence and basis for future follow-up studies that expand sample size. Although ISET cannot provide effective stratification for molecular typing and pathological staging of breast cancer, it is more affordable, easy to operate and homogeneous, which is more feasible to be transferred to the clinical settings for long-term follow up that requires repeated detection at multiple time points. Observations derived from this study have also provided some basis for the early monitoring of postoperative recurrence of breast cancer and the evaluation of the efficacy of systematic treatment by ISET.

\section{Acknowledgments}

Funding: This study was funded by the Natural Science Foundation of China (81572602).

\section{Footnote}

Reporting Checklist: The authors have completed the STROBE reporting checklist. Available at http://dx.doi. org/10.21037/tcr-19-2662

Data Sharing Statement: Available at http://dx.doi. org/10.21037/tcr-19-2662

Conflicts of Interest: All authors have completed the ICMJE uniform disclosure form (available at http://dx.doi. org/10.21037/tcr-19-2662). The authors have no conflicts of interest to declare.

Ethical Statement: The authors are accountable for all aspects of the work in ensuring that questions related to the accuracy or integrity of any part of the work are appropriately investigated and resolved. The trial was conducted in accordance with the Declaration of Helsinki (as revised in 2013). The study was approved by Institutional Review Boards of the First Affiliated Hospital with Nanjing Medical University (NO.: 2017-SR-171) and informed consent was taken from all the patients.

Open Access Statement: This is an Open Access article distributed in accordance with the Creative Commons Attribution-NonCommercial-NoDerivs 4.0 International License (CC BY-NC-ND 4.0), which permits the noncommercial replication and distribution of the article with the strict proviso that no changes or edits are made and the original work is properly cited (including links to both the 
formal publication through the relevant DOI and the license). See: https://creativecommons.org/licenses/by-nc-nd/4.0/.

\section{References}

1. Bray F, Ferlay J, Soerjomataram I, et al. Global cancer statistics 2018: GLO-BOCAN estimates of incidence and mortality worldwide for 36 cancers in 185 countries. CA Cancer J Clin 2018;68:394-424.

2. Fisher B, Jeong JH, Anderson S, et al. Twenty-fiveyear follow-up of a randomized trial comparing radical mastectomy, total mastectomy, and total mastectomy followed by irradiation. N Engl J Med 2002;347:567-75.

3. Pantel K, Alix-Panabieres C, Riethdorf S. Cancer micrometastases. Nat Rev Clin Oncol 2009;6:339-51.

4. Riethdorf S, Muller V, Zhang L, et al. Detection and HER2 expression of circulating tumor cells: prospective monitoring in breast cancer patients treated in the neoadjuvant GeparQuattro trial. Clin Cancer Res 2010;16:2634-45.

5. Pantel K, Brakenhoff RH, Brandt B. Detection, clinical relevance and specific biological properties of disseminating tumour cells. Nat Rev Cancer 2008;8:329-40.

6. Braun $S, \operatorname{Vogl} \mathrm{FD}$, Naume B, et al. A pooled analysis of bone marrow micrometastasis in breast cancer. $\mathrm{N}$ Engl J Med 2005;353:793-802.

7. Wiedswang G, Borgen E, Karesen R, et al. Detection of isolated tumor cells in bone marrow is an independent prognostic factor in breast cancer. J Clin Oncol 2003;21:3469-78.

8. Janni W, Rack B, Schindlbeck C, et al. The persistence of isolated tumor cells in bone marrow from patients with breast carcinoma predicts an increased risk for recurrence. Cancer 2005;103:884-91.

9. Thery L, Meddis A, Cabel L, et al. Circulating Tumor Cells in Early Breast Cancer. JNCI Cancer Spectr 2019;3:pkz026.

10. Bidard FC, Proudhon C, Pierga JY. Circulating tumor cells in breast cancer. Mol Oncol 2016;10:418-30.

11. Ignatiadis $M$, Lee $M$, Jeffrey SS. Circulating Tumor Cells and Circulating Tumor DNA: Challenges and Opportunities on the Path to Clinical Utility. Clin Cancer Res 2015;21:4786-800.

12. Park HS, Han HJ, Lee S, et al. Detection of Circulating Tumor Cells in Breast Cancer Pa-tients Using Cytokeratin-19 Real-Time RT-PCR. Yonsei Med J 2017;58:19-26.

13. Andree KC, van Dalum G, Terstappen LW. Challenges in circulating tumor cell detection by the CellSearch system. Mol Oncol 2016;10:395-407.

14. Yoneda K, Chikaishi Y, Kuwata T, et al. Capture of mesothelioma cells with 'universal' CTC-chip. Oncol Lett 2018;15:2635-40.

15. Danila DC, Samoila A, Patel C, et al. Clinical Validity of Detecting Circulating Tumor Cells by AdnaTest Assay Compared With Direct Detection of Tumor mRNA in Stabilized Whole Blood, as a Biomarker Predicting Overall Survival for Metastatic Castration-Resistant Prostate Cancer Patients. Cancer J 2016;22:315-20.

16. Huang Q, Wang FB, Yuan CH, et al. Gelatin Nanoparticle-Coated Silicon Beads for Density-Selective Capture and Release of Heterogeneous Circulating Tumor Cells with High Purity. Theranostics 2018;8:1624-35.

17. Vona G, Sabile A, Louha M, et al. Isolation by size of epithelial tumor cells : a new method for the immunomorphological and molecular characterization of circulatingtumor cells. Am J Pathol 2000;156:57-63.

18. Dementeva N, Kokova D, Mayboroda OA. Current Methods of the Circulating Tumor Cells (CTC) Analysis: A Brief Overview. Curr Pharm Des 2017;23:4726-8.

19. de Wit S, Zeune LL, Hiltermann TJN, et al. Classification of Cells in CTC-Enriched Samples by Advanced Image Analysis. Cancers (Basel) 2018;10:377.

20. Riethdorf S, O'Flaherty L, Hille C, et al. Clinical applications of the CellSearch platform in cancer patients. Adv Drug Deliv Rev 2018;125:102-21.

21. Neurauter AA, Bonyhadi M, Lien E, et al. Cell isolation and expansion using Dynabeads. Adv Biochem Eng Biotechnol 2007;106:41-73.

22. Adams DL, Stefansson S, Haudenschild C, et al. Cytometric characterization of circulating tumor cells captured by microfiltration and their correlation to the CellSearch((R)) CTC test. Cytometry A 2015;87:137-44.

23. Nicolazzo C, Raimondi C, Francescangeli F, et al. EpCAM-expressing circulating tumor cells in colorectal cancer. Int J Biol Markers 2017;32:e415-20.

24. Rao CG, Chianese D, Doyle GV, et al. Expression of epithelial cell adhesion molecule in carcinoma cells present in blood and primary and metastatic tumors. Int $\mathrm{J}$ Oncol 2005;27:49-57.

25. Alunni-Fabbroni M, Sandri MT. Circulating tumour cells in clinical practice: Methods of detection and possible characterization. Methods 2010;50:289-97.

26. Gertler R, Rosenberg R, Fuehrer K, et al. Detection of circulating tumor cells in blood using an optimized density gradient centrifugation. Recent Results Cancer Res 
2003;162:149-55.

27. Müller V, Stahmann N, Riethdorf S, et al. Circulating tumor cells in breast cancer: corre-lation to bone marrow micrometastases, heterogeneous response to systemic therapy and low proliferative activity. Clin Cancer Res 2005;11:3678-85.

28. Andergassen U, Kolbl AC, Mahner S, et al. Real-time RTPCR systems for CTC detection from blood samples of breast cancer and gynaecological tumour patients (Review). Oncol Rep 2016;35:1905-15.

29. Hofman VJ, Ilie MI, Bonnetaud C, et al. Cytopathologic detection of circulating tumor cells using the isolation by size of epithelial tumor cell method: promises and pitfalls. Am J Clin Pathol 2011;135:146-56.

30. Ried K, Eng P, Sali A. Screening for Circulating Tumour Cells Allows Early Detection of Cancer and Monitoring of Treatment Effectiveness: An Observational Study. Asian Pac J Cancer Prev 2017;18:2275-85.

Cite this article as: Zeng $\mathrm{H}$, Veeramootoo JS, Ma G, Jiang Y, Wang J, Xia T, Liu X. Clinical value and feasibility of ISET in detecting circulating tumor cells in early breast cancer. Transl Cancer Res 2020;9(7):4297-4305. doi: 10.21037/tcr-19-2662
31. Castle J, Morris K, Pritchard S, et al. Challenges in enumeration of CTCs in breast cancer using techniques independent of cytokeratin expression. PLoS One 2017;12:e0175647.

32. Zhao Y, Zhao S, Chen Y, et al. Isolation of circulating tumor cells in patients undergoing surgery for esophageal cancer and a specific confirmation method. Oncol Lett 2019;17:3817-25.

33. Hofman V, Ilie MI, Long E, et al. Detection of circulating tumor cells as a prognostic factor in patients undergoing radical surgery for non-small-cell lung carcinoma: comparison of the efficacy of the CellSearch Assay and the isolation by size of epithelial tumor cell method. Int J Cancer 2011;129:1651-60.

34. Bidard FC, Mathiot C, Delaloge S, et al. Single circulating tumor cell detection and overall survival in nonmetastatic breast cancer. Ann Oncol 2010;21:729-33. 